\title{
Fruit, vegetable and bean intake and mortality from cardiovascular disease among Japanese men and women: the JACC Study
}

\author{
Junko Nagura $^{1}$, Hiroyasu Iso ${ }^{2}$, Yoshiyuki Watanabe ${ }^{1}$, Koutatsu Maruyama ${ }^{2}$, Chigusa Date $^{3}$, \\ Hideaki Toyoshima ${ }^{4}$, Akio Yamamoto ${ }^{5}$, Shogo Kikuchi ${ }^{6}$, Akio Koizumi ${ }^{7}$, Takaaki Kondo ${ }^{4}$, \\ Yasuhiko $\mathrm{Wada}^{8}$, Yutaka Inaba ${ }^{9}$, Akiko Tamakoshi ${ }^{6}$ and the JACC Study group \\ ${ }^{1}$ Department of Social Medicine \& Cultural Sciences, Research Institute for Neurological Diseases \& Geriatrics, Kyoto Prefectural \\ University of Medicine, Kyoto, Japan \\ ${ }^{2}$ Public Health, Department of Social and Environmental Medicine, Graduate School of Medicine, Osaka University, 2-2 \\ Yamadaoka, Shuita-shi, Osaka 565-0871, Japan \\ ${ }^{3}$ Faculty of Human Life and Environment, Nara Women's University, Nara 630-8506, Japan \\ ${ }^{4}$ Department of Public Health/Health Information Dynamics, Fields of Science, Program of Health and Community Medicine, \\ Nagoya University Graduate School of Medicine, Nagoya 466-8550, Japan \\ ${ }^{5}$ Infectious Disease Research Division, Infectious Disease Surveillance Center, Hyogo Prefectural Institute of Public Health and \\ Environmental Sciences, Hyogo 652-0032, Japan \\ ${ }^{6}$ Department of Public Health, Aichi Medical University, Aichi 480-1195, Japan \\ ${ }^{7}$ Department of Health and Environmental Sciences, Graduate School of Medicine, Kyoto University, Kyoto 606-8501, Japan \\ ${ }^{8}$ Department of Medical Informatics and Occupational Epidemiology, Japan Labour Health and Welfare Organization, Kansai \\ Rosai Hospital, Hyogo 660-8511, Japan \\ ${ }^{9}$ Department of Epidemiology and Environmental Health, School of Medicine, Juntendo University, Tokyo 113-8421, Japan
}

(Received 16 April 2008 - Revised 2 September 2008 - Accepted 13 October 2008 - First published online 13 January 2009)

To examine the association of plant-based food intakes with CVD and total mortality among Japanese. In the Japan Collaborative Cohort Study for Evaluation of Cancer Risk, 25206 men and 34279 women aged 40-79 years, whose fruit, vegetable and bean intakes were assessed by questionnaire at baseline in 1988-90, were followed for 13 years. Deaths from total stroke, stroke subtypes, CHD and total CVD, according to the International Classification for Diseases 10th Revision, were registered. During 756054 person-years of follow-up, there were 559 deaths from total stroke, 258 from CHD, 1207 from total CVD and 4514 from total mortality for men, and for women, 494, 194, 1036 and 3092 , respectively. Fruit intake was inversely associated with mortality from total stroke (the multivariable hazard ratio (HR (95\% CI)) in the highest $v$. lowest quartiles $=0.67(0.55,0.81))$, total CVD $(\mathrm{HR}=0.75(0.66,0.85))$ and total mortality $(\mathrm{HR}=0.86(0.80,0.92))$. Vegetable intake was inversely associated with total CVD $(\mathrm{HR}=0.88(0.78,0.99))$. Bean intake was inversely associated with other CVD $(\mathrm{HR}=0.79(0.64,0.98))$, total $\mathrm{CVD}(\mathrm{HR}=0.84(0.74,0.95))$ and total mortality $(\mathrm{HR}=0.90(0.84,0.96))$. Further adjustment for other plant-based foods did not alter the association of fruit intake with mortality from total stroke, total CVD and total mortality, but attenuated the associations of vegetables and beans with mortality risk. In conclusion, intakes of plant-based foods, particularly fruit intake, were associated with reduced mortality from CVD and all causes among Japanese men and women.

Fruits: Vegetables: Beans: CVD: Mortality

Protective effects of plant-based foods against CVD have been suggested by prospective cohort studies in Western countries $^{(1-6)}$. Fruit and vegetable intakes were associated with reduced risks of stroke and $\mathrm{CHD}^{(1,2)}$, and nut intake was associated with a reduced risk of $\mathrm{CHD}^{(3-6)}$. These potential effects of plant-based foods need to be examined for Japanese, because of their different profiles of CVD and diet. In Japan, the incidence of stroke is higher than that of CHD, and the proportion of haemorrhagic stroke among the stroke subtypes is higher than in Western countries ${ }^{(7,8)}$. The Japanese habitually consume more beans than the Westerners, and soyabeans, in particular, have recently been highlighted as a protective factor for CVD in Western countries ${ }^{(9,10)}$.

So far, several Japanese studies have shown inverse associations of the fruit, vegetable or bean intake with the risk of stroke $^{(11-14)}$. The Hiroshima/Nagasaki Life Span Study showed a protective association of both fruit and vegetable intakes with mortality from both ischaemic stroke and intra-parenchymal haemorrhage ${ }^{(11)}$. The Shibata Study showed a protective association of vegetable intake with the incidence of total stroke ${ }^{(12)}$.

Abbreviation: HR, hazard ratio.

* Corresponding author: Professor Hiroyasu Iso, fax +8166879 3919, email iso@pbhel.med.osaka-u.ac.jp 
The Japan Public Health Center-based Prospective Study showed a protective association of fruit intake with the incidence of CVD for Japanese men and women combined ${ }^{(13)}$, and also showed a protective association of soya intake with the incidence of cerebral infarction and mortality from CVD for women ${ }^{(14)}$. The Takayama Study also showed a protective association of vegetable intake with mortality from CVD for Japanese women ${ }^{(15)}$ The Japan Collaborative Cohort Study for Evaluation of Cancer Risk previously reported the association between plant-based food intakes and mortality from CVD, cancer and all causes ${ }^{(16)}$, but these associations were not adjusted for major confounding factors.

Therefore, no study has examined whether plant-based foods were associated with CVD, their subtypes and total mortality systematically in Japan. Such a study in Japanese is also of value because plant-based food intakes are positively correlated with saturated fat intake in Japanese ${ }^{(15)}$ unlike Western populations ${ }^{(17,18)}$, and confounding factors may be different from Western studies. We hypothesised that higher plant-based food intake had beneficial effects for the prevention of CVD and their subtypes in general Japanese populations, and we comprehensively examined the associations of the fruit, vegetable and bean intake with mortality from stroke, stroke subtypes, CHD, total CVD and all causes in a 13-year cohort study of approximately 60000 Japanese men and women.

\section{Experimental methods}

\section{Subjects}

The Japan Collaborative Cohort Study sponsored by Monbusho, the Ministry of Education, Science, Sports and Culture, began in 1988-90 when 110792 individuals (46465 men and 64327 women) aged 40-79 years living in forty-five communities across Japan participated in municipal health screening examinations and completed a self-administered questionnaire about their lifestyles (habits of smoking and drinking, physical activity, hours of sleep, education and mental stress) and medical histories (hypertension, diabetes, CVD and cancer). Informed consent was obtained before completing the questionnaire. We excluded 2576 men and 3288 women from the analysis because of previous history of stroke, $\mathrm{CHD}$ or cancer at baseline. Persons (18683 men and 26760 women) with missing information regarding the intake of fruits, vegetables and beans were also excluded, and a total of 25206 men and 34279 women were used for the analysis. There was no substantial difference in mortality rates between persons who gave the valid dietary information and those who did not; the multivariable hazard ratios (HR $(95 \% \mathrm{CI}))$ for respondents $v$. non-respondents were 1.08 $(0.98,1.21)$ for total stroke, $0.98(0.83,1.14)$ for CHD, 1.04 $(0.97,1.12)$ for total CVD and $1.02(0.98,1.07)$ for all causes. No material differences were also found between the respondents and non-respondents for BMI, history of hypertension, history of diabetes, smoking, ethanol intake and other cardiovascular risk characteristics.

\section{Dietary assessment}

The self-administered FFQ was conducted to estimate the consumption of thirty-three foods during the past year ${ }^{(19)}$.
The food items were beef, pork, ham or sausage, chicken, liver, eggs, milk, yogurt, cheese, butter, margarine, deep-fried foods or tempura, fried vegetables, fresh fish, steamed fish paste, dried fish or salted fish, spinach or garland chrysanthemum, carrot or pumpkin, tomatoes, cabbage or head lettuce, Chinese cabbage, edible wild plants, fungi, potatoes, algae, pickles, preserved foods using soya sauce, boiled beans, tofu, citrus fruits, fruits excluding citrus varieties, fresh fruit juice in summer and sweets. Each food had a five-level precoded answer: 'rarely eat'; 'once or twice per month'; 'once or twice per week'; 'three or four times per week'; 'almost daily'. Then, we converted the answers 'rarely eat' to 0 , 'once or twice per month' to $0 \cdot 375$, 'once or twice per week' to 1.5 , 'three or four times per week' to 3.5 and 'almost daily' to 7 servings per $d$ to estimate the average weekly intake of each fruit (citrus fruits, fruits excluding citrus varieties and fresh fruit juice in summer), vegetable (spinach or garland chrysanthemum, carrot or pumpkin, tomatoes, cabbage or head lettuce and Chinese cabbage) and beans (tofu, i.e. soyabean curd, and boiled beans) for each participant. The average weekly intakes of individual foods were combined to compute the total fruit, vegetable and bean intakes.

The reproducibility of the dietary data was confirmed by comparing two questionnaires administered 1 year apart for eightyfive subjects (eight men and seventy-seven women) ${ }^{(19)}$. The median (range) values of the Spearman correlation coefficients were $0.57(0.55,0.58)$ for three items of fruits, $0.63(0.43$, $0.66)$ for five items of vegetables and $0.62(0.59,0.64)$ for two items of beans. The validity of the data was confirmed by comparing the data from the questionnaire with those from four 3-d dietary records for the eighty-five subjects, collected approximately 3-4 months apart ${ }^{(19)}$. The median values of the Spearman correlation coefficients were $0.26(0.24,0.39)$ for three items of fruits, $0.33(0 \cdot 18,0.45)$ for five items of vegetables and $0.40(0.30,0.50)$ for two items of beans. The intakes of selective nutrients, i.e. cholesterol, saturated, $n-3$ polyunsaturated and sodium intake, were calculated and adjusted using the residual method, and used as potential confounding factors for the analysis.

\section{Mortality surveillance}

For mortality surveillance in each community, investigators systematically reviewed death certificates, all of which were filed in the public-health centre in the area of residency. Mortality data were sent centrally to the Ministry of Health and Welfare and the underlying causes of deaths were coded for the National Vital Statistics according to the International Classification for Diseases, 9th Revision from 1988 to 1994 and 10th Revision from 1995 to 2003. Registration of death is required by the Family Registration Law in Japan, and is believed to be completed across the country. Therefore, all deaths that occurred in the cohort were ascertained by death certificates from the public-health centres, except for subjects who died after they moved from their original community, in which case the subject was treated as censored. The follow-up was conducted until the end of 2003 and the average follow-up period for the participants was 12.7 years.

Cause-specific mortality was defined separately for total stroke (International Classification for Diseases-9 codes 
Table 1. Age- and sex-adjusted mean values or prevalence of cardiovascular risk factors according to quartiles of the frequency of the fruit, vegetable and bean intakes*

\begin{tabular}{|c|c|c|c|c|c|c|c|c|c|c|c|c|c|c|c|}
\hline & \multicolumn{4}{|c|}{ Quartiles of fruit intake } & \multirow{2}{*}{$\begin{array}{l}P \text { for } \\
\text { trend }\end{array}$} & \multicolumn{4}{|c|}{ Quartiles of vegetable intake } & \multirow{2}{*}{$\begin{array}{l}P \text { for } \\
\text { trend }\end{array}$} & \multicolumn{4}{|c|}{ Quartiles of bean intake } & \multirow{2}{*}{$\begin{array}{l}P \text { for } \\
\text { trend }\end{array}$} \\
\hline & Q1 & Q2 & Q3 & Q4 & & Q1 & Q2 & Q3 & Q4 & & Q1 & Q2 & Q3 & Q4 & \\
\hline Servings per week & 0.9 & $2 \cdot 3$ & 3.9 & 5.9 & - & $1 \cdot 2$ & $2 \cdot 3$ & 3.4 & $5 \cdot 2$ & - & 0.8 & $1 \cdot 8$ & 3.0 & 4.5 & - \\
\hline Number of subjects & 14967 & 14066 & 17607 & 12845 & - & 14768 & 15213 & 14142 & 15362 & - & 15212 & 15573 & 12321 & 16379 & - \\
\hline Age (years) & 55.9 & 56.4 & 56.6 & 56.1 & $<0.001$ & $55 \cdot 3$ & 55.5 & 56.4 & 57.8 & $<0.001$ & 54.9 & 55.5 & $57 \cdot 0$ & 57.7 & $<0.001$ \\
\hline Women (\%) & 43.8 & $55 \cdot 6$ & $63 \cdot 3$ & $68 \cdot 2$ & $<0.001$ & $46 \cdot 3$ & 56.8 & $61 \cdot 4$ & 65.9 & $<0.001$ & 51.5 & $56 \cdot 3$ & $57 \cdot 3$ & 64.7 & $<0.001$ \\
\hline BMI $\left(\mathrm{kg} / \mathrm{m}^{2}\right)$ & $22 \cdot 8$ & $22 \cdot 8$ & $22 \cdot 8$ & 22.9 & 0.07 & 22.9 & 22.8 & $22 \cdot 8$ & 22.9 & 0.16 & $22 \cdot 8$ & $22 \cdot 8$ & $22 \cdot 8$ & 22.9 & 0.24 \\
\hline $\begin{array}{l}\text { History of } \\
\text { hypertension (\%) }\end{array}$ & 20.4 & $20 \cdot 0$ & $19 \cdot 6$ & 19.5 & 0.04 & $20 \cdot 0$ & $20 \cdot 3$ & $19 \cdot 8$ & 19.4 & 0.08 & $19 \cdot 9$ & $20 \cdot 7$ & $20 \cdot 0$ & $19 \cdot 1$ & 0.01 \\
\hline History of diabetes (\%) & 4.9 & 4.9 & 4.7 & 3.9 & $<0.001$ & 4.4 & 4.6 & 4.7 & 4.7 & 0.29 & 4.5 & 4.7 & 4.6 & 4.6 & 0.95 \\
\hline Current smoking (\%) & 31.1 & $26 \cdot 3$ & $24 \cdot 7$ & 23.7 & $<0.001$ & $30 \cdot 1$ & $26 \cdot 1$ & $25 \cdot 4$ & 24.4 & $<0.001$ & 29.5 & $26 \cdot 9$ & 25.8 & 23.8 & 0.14 \\
\hline Ethanol intake $(\mathrm{g} / \mathrm{d})$ & 31.1 & $27 \cdot 6$ & $26 \cdot 9$ & $26 \cdot 4$ & $<0.001$ & $29 \cdot 3$ & $28 \cdot 1$ & $28 \cdot 2$ & 27.6 & $<0.001$ & 28.6 & 28.4 & 28.4 & $28 \cdot 0$ & $<0.001$ \\
\hline $\begin{array}{l}\text { Walk } 30 \text { min or more/ } \\
\text { week (\%) }\end{array}$ & $68 \cdot 3$ & 69.9 & $71 \cdot 2$ & $73 \cdot 2$ & $<0.001$ & $65 \cdot 8$ & $70 \cdot 6$ & $72 \cdot 1$ & 74.1 & $<0.001$ & 68.6 & $70 \cdot 2$ & $72 \cdot 2$ & $71 \cdot 9$ & $<0.001$ \\
\hline $\begin{array}{l}\text { Sports } 1 \mathrm{~h} \text { or more/ } \\
\text { week (\%) }\end{array}$ & $22 \cdot 1$ & $25 \cdot 2$ & 29.1 & 30.5 & $<0.001$ & $22 \cdot 5$ & $25 \cdot 8$ & 27.8 & 30.7 & $<0.001$ & 23.9 & 25.8 & 27.8 & 29.4 & $<0.001$ \\
\hline Hours of sleep $(\mathrm{h} / \mathrm{d})$ & $7 \cdot 3$ & $7 \cdot 2$ & $7 \cdot 2$ & $7 \cdot 2$ & 0.01 & $7 \cdot 2$ & $7 \cdot 2$ & $7 \cdot 2$ & $7 \cdot 3$ & $<0.001$ & $7 \cdot 2$ & $7 \cdot 2$ & $7 \cdot 2$ & $7 \cdot 3$ & $<0.001$ \\
\hline $\begin{array}{l}\text { College or higher } \\
\text { education (\%) }\end{array}$ & $10 \cdot 4$ & $12 \cdot 2$ & $14 \cdot 6$ & $16 \cdot 3$ & $<0.001$ & 11.4 & $13 \cdot 1$ & 13.5 & $15 \cdot 4$ & $<0.001$ & $12 \cdot 3$ & $12 \cdot 9$ & $14 \cdot 0$ & $14 \cdot 3$ & $<0.001$ \\
\hline $\begin{array}{l}\text { High perceived mental } \\
\text { stress (\%) }\end{array}$ & $22 \cdot 3$ & $22 \cdot 4$ & $22 \cdot 2$ & $23 \cdot 1$ & 0.23 & 23.5 & $22 \cdot 4$ & $22 \cdot 0$ & $22 \cdot 0$ & 0.004 & $23 \cdot 2$ & $22 \cdot 3$ & $22 \cdot 4$ & $22 \cdot 0$ & 0.04 \\
\hline $\begin{array}{l}\text { Total energy intake } \\
(\mathrm{kJ})\end{array}$ & 5640 & 5933 & 6243 & 6636 & $<0.001$ & 5427 & 5958 & 6272 & 6728 & $<0.001$ & 5498 & 5929 & 6314 & 6669 & $<0.001$ \\
\hline Cholesterol intake $(\mathrm{mg})$ & 219 & 237 & 250 & 258 & $<0.001$ & 208 & 233 & 250 & 271 & $<0.001$ & 215 & 235 & 250 & 264 & $<0.001$ \\
\hline SFA intake (g) & 8.4 & 9.0 & 9.5 & 9.9 & $<0.001$ & $8 \cdot 2$ & $9 \cdot 0$ & 9.4 & $10 \cdot 0$ & $<0.001$ & 8.5 & $9 \cdot 0$ & 9.5 & 9.8 & $<0.001$ \\
\hline $\begin{array}{l}n-3 \text { Fatty acid } \\
\text { intake }(\mathrm{g})\end{array}$ & 1.5 & 1.6 & $1 \cdot 7$ & $1 \cdot 8$ & $<0.001$ & 1.4 & 1.6 & $1 \cdot 7$ & 1.9 & $<0.001$ & 1.4 & 1.6 & 1.7 & 1.9 & $<0.001$ \\
\hline Sodium intake (mg) & 2065 & 2110 & 2141 & 2167 & $<0.001$ & 1902 & 2058 & 2166 & 2345 & $<0.001$ & 1929 & 2057 & 2175 & 2316 & $<0.001$ \\
\hline
\end{tabular}


Table 2. Risk of mortality from stroke, $\mathrm{CHD}$, total CVD and all causes according to quartiles of the frequency of fruit intake (Hazard ratio (HR) values and $95 \% \mathrm{Cl}$ )

\begin{tabular}{|c|c|c|c|c|c|c|c|c|}
\hline & \multicolumn{7}{|c|}{ Quartiles of fruit intake } & \multirow[b]{3}{*}{$P$ for trend } \\
\hline & \multirow[b]{2}{*}{ Q1 } & \multicolumn{2}{|r|}{ Q2 } & \multicolumn{2}{|r|}{ Q3 } & \multicolumn{2}{|c|}{ Q4 } & \\
\hline & & $\mathrm{HR}$ & $95 \% \mathrm{Cl}$ & $\mathrm{HR}$ & $95 \% \mathrm{Cl}$ & $\mathrm{HR}$ & $95 \% \mathrm{Cl}$ & \\
\hline Person-years & 187700 & \multicolumn{2}{|r|}{178625} & \multicolumn{2}{|r|}{223683} & \multicolumn{2}{|c|}{166046} & \\
\hline \multicolumn{9}{|l|}{ Total stroke } \\
\hline Number & 348 & \multicolumn{2}{|r|}{258} & \multicolumn{2}{|r|}{284} & \multicolumn{2}{|c|}{163} & \\
\hline Age- and sex-adjusted HR & 1.00 & 0.77 & $0.66,0.91$ & 0.68 & $0.58,0.80$ & 0.57 & $0.48,0.69$ & $<0.001$ \\
\hline Multivariable $\mathrm{HR}^{*}$ & 1.00 & 0.83 & $0.71,0.98$ & 0.79 & $0.67,0.92$ & 0.67 & $0.55,0.81$ & $<0.001$ \\
\hline Multivariable HR† & 1.00 & 0.81 & $0.69,0.96$ & 0.76 & $0.64,0.90$ & 0.65 & $0.53,0.80$ & $<0.001$ \\
\hline \multicolumn{9}{|l|}{ Haemorrhagic stroke } \\
\hline Number & 130 & \multicolumn{2}{|r|}{93} & \multicolumn{2}{|r|}{108} & \multicolumn{2}{|c|}{62} & \\
\hline Age- and sex-adjusted HR & 1.00 & 0.73 & $0.56,0.96$ & 0.67 & $0.52,0.87$ & 0.55 & $0.40,0.74$ & $<0.001$ \\
\hline Multivariable $\mathrm{HR}^{*}$ & 1.00 & 0.79 & $0.60,1.03$ & 0.76 & $0.59,0.99$ & 0.63 & $0.46,0.87$ & 0.004 \\
\hline Multivariable HR† & 1.00 & 0.76 & $0.58,1.00$ & 0.72 & $0.55,0.95$ & 0.59 & $0.42,0.82$ & 0.002 \\
\hline \multicolumn{9}{|l|}{ Ischaemic stroke } \\
\hline Number & 121 & \multicolumn{2}{|r|}{82} & \multicolumn{2}{|r|}{102} & \multicolumn{2}{|c|}{57} & \\
\hline Age- and sex-adjusted HR & 1.00 & 0.71 & $0.54,0.95$ & 0.72 & $0.55,0.94$ & 0.60 & $0.44,0.82$ & 0.002 \\
\hline Multivariable $\mathrm{HR}^{*}$ & 1.00 & 0.77 & $0.58,1.03$ & 0.85 & $0.65,1.12$ & 0.72 & $0.52,1.00$ & 0.070 \\
\hline Multivariable HR† & 1.00 & 0.76 & $0.57,1.01$ & 0.83 & $0.63,1.11$ & 0.71 & $0.50,1.00$ & 0.081 \\
\hline \multicolumn{9}{|l|}{$\mathrm{CHD}$} \\
\hline Number & 146 & \multicolumn{2}{|r|}{116} & \multicolumn{2}{|r|}{117} & \multicolumn{2}{|c|}{73} & \\
\hline Age- and sex-adjusted HR & 1.00 & 0.84 & $0.66,1.07$ & 0.69 & $0.54,0.88$ & 0.63 & $0.47,0.83$ & $<0.001$ \\
\hline Multivariable $\mathrm{HR}^{*}$ & 1.00 & 0.92 & $0.72,1.18$ & 0.79 & $0.62,1.02$ & 0.74 & $0.55,0.99$ & 0.015 \\
\hline Multivariable HR† & 1.00 & 0.97 & $0.75,1.24$ & 0.84 & $0.65,1.10$ & 0.79 & $0.58,1.08$ & 0.061 \\
\hline Other CVD & & & & & & & & \\
\hline Number & 205 & & 173 & & 229 & & 31 & \\
\hline Age- and sex-adjusted HR & 1.00 & 0.88 & $0.72,1.08$ & 0.94 & $0.78,1.14$ & 0.78 & $0.63,0.98$ & 0.060 \\
\hline Multivariable $\mathrm{HR}^{\star}$ & 1.00 & 0.95 & $0.77,1.17$ & 1.06 & $0.87,1.29$ & 0.89 & $0.71,1.12$ & 0.553 \\
\hline Multivariable HR† & 1.00 & 0.99 & $0.80,1.22$ & $1 \cdot 13$ & $0.92,1.38$ & 0.96 & $0.76,1.23$ & 0.988 \\
\hline Total CVD & & & & & & & & \\
\hline Number & 699 & & 547 & & 630 & & 67 & \\
\hline Age- and sex-adjusted HR & 1.00 & $0 \cdot 82$ & $0.73,0.91$ & 0.76 & $0.68,0.85$ & 0.65 & $0.57,0.73$ & $<0.001$ \\
\hline Multivariable $\mathrm{HR}^{*}$ & 1.00 & 0.88 & $0.79,0.99$ & 0.87 & $0.78,0.97$ & 0.75 & $0.66,0.85$ & $<0.001$ \\
\hline Multivariable HR $\dagger$ & 1.00 & 0.90 & $0.80,1.00$ & 0.89 & $0.79,0.99$ & 0.77 & $0.67,0.88$ & $<0.001$ \\
\hline All causes & & & & & & & & \\
\hline Number & 2284 & & 1824 & & 2158 & & 40 & \\
\hline Age- and sex-adjusted HR & 1.00 & 0.86 & $0.81,0.91$ & 0.83 & $0.79,0.89$ & 0.76 & $0.71,0.81$ & $<0.001$ \\
\hline Multivariable $\mathrm{HR}^{\star}$ & 1.00 & 0.91 & $0.86,0.97$ & 0.93 & $0.87,0.99$ & 0.86 & $0.80,0.92$ & $<0.001$ \\
\hline Multivariable HR† & 1.00 & 0.92 & $0.86,0.98$ & 0.93 & $0.87,0.99$ & 0.86 & $0.80,0.93$ & $<0.001$ \\
\hline
\end{tabular}

*Adjusted for sex, age, BMI, smoking status, alcohol intake, hours of walking, hours of sleep, education years, perceived mental stress, cholesterol intake, SFA intake, $n$ - 3 fatty acids intake, sodium intake and histories of hypertension and diabetes.

† Adjusted further for vegetable and bean intakes.

430-438 and International Classification for Diseases-10 codes I60-I69), CHD (410-414 and I20-I25), other CVD (390-409, 415-429, 439-459, I01-I19, I26-I59 and I70-I99) and total CVD (390-459 and I01-I99). Total stroke was further divided into haemorrhagic stroke (430431 and I60-61) and ischaemic stroke (433-434 and I63). Total mortality was also examined as a reference. The present study was approved by the Ethical Committee, the Nagoya University School of Medicine and the University of Tsukuba.

\section{Statistical analysis}

Statistical analyses were based on sex-specific mortality during the follow-up period from 1989 to 2003. For each participant, the person-year of follow-up was calculated when they died or moved out of his or her community or the end of 2003, whichever was the first. The age- and sex-adjusted risk of mortality from CVD as well as total mortality was defined as the corresponding death rate among the participants according to quartiles of the fruit, vegetable and bean intakes.
The means and proportions of selected cardiovascular risk factors were calculated according to quartiles of those food intakes. We calculated the quartile cut-points among the whole study population and used the lowest quartiles as the reference categories for the analyses of relative risk for the second, third and highest quartiles. The HR and their $95 \%$ CI were calculated after adjustment for age, sex and potential confounding factors using the Cox proportional hazard model. These confounding variables, which were associated with CVD among Japanese, included BMI (sexspecific quintiles), smoking category (never, ex- and current smokers of $\leq 19$ or $\geq 20$ cigarettes per d), alcohol intake category (never, ex- and current ethanol intake of 1-22, 23-45, $46-68$ and $\geq 69 \mathrm{~g} / \mathrm{d}$ ), hours of walking (rarely, 30, 30-60 and $\geq 60$ min per $\mathrm{d}$ ), sports ( $<1$ and $\geq 1 \mathrm{~h}$ per week), education $(<10,10-12,13-15$ and $\geq 16$ years $)$, perceived mental stress (low, medium and high), history of hypertension or diabetes and sex-specific quartiles of dietary cholesterol, SFA, $n-3$ PUFA and sodium intake (sex-specific quartiles). Since the plant-based foods have little of those nutrients, the 
Table 3. Risk of mortality from stroke, CHD, total CVD and all causes according to quartiles of the frequency of vegetable intake (Hazard ratio (HR) values and $95 \% \mathrm{Cl}$ )

\begin{tabular}{|c|c|c|c|c|c|c|c|c|}
\hline & \multicolumn{7}{|c|}{ Quartiles of vegetable intake } & \multirow[b]{3}{*}{$P$ for trend } \\
\hline & \multirow[b]{2}{*}{ Q1 } & \multicolumn{2}{|r|}{ Q2 } & \multicolumn{2}{|r|}{ Q3 } & \multicolumn{2}{|c|}{ Q4 } & \\
\hline & & $\mathrm{HR}$ & $95 \% \mathrm{Cl}$ & $\mathrm{HR}$ & $95 \% \mathrm{Cl}$ & $\mathrm{HR}$ & $95 \% \mathrm{Cl}$ & \\
\hline Person-years & 185787 & \multicolumn{2}{|r|}{193546} & \multicolumn{2}{|r|}{180543} & \multicolumn{2}{|c|}{196177} & \\
\hline \multicolumn{9}{|l|}{ Total stroke } \\
\hline Number & 258 & \multicolumn{2}{|r|}{245} & \multicolumn{2}{|r|}{254} & \multicolumn{2}{|c|}{296} & \\
\hline Age- and sex-adjusted HR & 1.00 & 0.93 & $0.78,1 \cdot 10$ & 0.97 & $0.81,1.15$ & 0.91 & $0.77,1.08$ & 0.349 \\
\hline Multivariable $\mathrm{HR}^{\star}$ & 1.00 & 0.97 & $0.82,1.16$ & 1.04 & $0.87,1.24$ & 0.97 & $0.81,1.16$ & 0.790 \\
\hline Multivariable HR† & 1.00 & 1.02 & $0.85,1.22$ & $1 \cdot 11$ & $0.92,1.34$ & 1.09 & $0.90,1.33$ & 0.256 \\
\hline \multicolumn{9}{|l|}{ Haemorrhagic stroke } \\
\hline Number & 98 & \multicolumn{2}{|r|}{101} & \multicolumn{2}{|r|}{76} & \multicolumn{2}{|c|}{118} & \\
\hline Age- and sex-adjusted HR & 1.00 & 0.99 & $0.75,1.31$ & 0.76 & $0.56,1.02$ & 0.98 & $0.75,1.28$ & 0.720 \\
\hline Multivariable $\mathrm{HR}^{\star}$ & 1.00 & 1.06 & $0.80,1.40$ & 0.84 & $0.62,1.14$ & $1 \cdot 10$ & $0.82,1.48$ & 0.638 \\
\hline Multivariable $\mathrm{HR} \dagger$ & 1.00 & 1.09 & $0.82,1.45$ & 0.88 & $0.64,1.21$ & $1 \cdot 22$ & $0.89,1.66$ & 0.235 \\
\hline \multicolumn{9}{|l|}{ Ischaemic stroke } \\
\hline Number & 92 & \multicolumn{2}{|r|}{74} & \multicolumn{2}{|r|}{98} & \multicolumn{2}{|c|}{98} & \\
\hline Age- and sex-adjusted HR & 1.00 & 0.79 & $0.58,1.07$ & 1.05 & $0.79,1.39$ & 0.84 & $0.63,1.12$ & 0.492 \\
\hline Multivariable $\mathrm{HR}^{\star}$ & 1.00 & 0.83 & $0.61,1.13$ & $1 \cdot 14$ & $0.85,1.54$ & 0.91 & $0.67,1.24$ & 0.884 \\
\hline Multivariable HR† & 1.00 & 0.87 & $0.64,1.20$ & 1.24 & $0.91,1.70$ & 1.03 & $0.74,1.43$ & 0.591 \\
\hline \multicolumn{9}{|l|}{$\mathrm{CHD}$} \\
\hline Number & 140 & \multicolumn{2}{|r|}{105} & \multicolumn{2}{|r|}{96} & \multicolumn{2}{|c|}{111} & \\
\hline Age- and sex-adjusted HR & 1.00 & 0.74 & $0.57,0.95$ & 0.69 & $0.53,0.89$ & 0.65 & $0.51,0.84$ & 0.002 \\
\hline Multivariable $\mathrm{HR}^{\star}$ & 1.00 & 0.79 & $0.61,1.02$ & 0.78 & $0.60,1.02$ & 0.77 & $0.58,1.00$ & 0.079 \\
\hline Multivariable HR† & 1.00 & 0.82 & $0.63,1.07$ & 0.83 & $0.63,1.10$ & 0.85 & $0.64,1.14$ & 0.376 \\
\hline Other CVD & & & & & & & & \\
\hline Number & 207 & & 176 & & 156 & & 99 & \\
\hline Age- and sex-adjusted HR & 1.00 & 0.83 & $0.68,1.01$ & 0.74 & $0.60,0.91$ & 0.76 & $0.62,0.92$ & 0.067 \\
\hline Multivariable $\mathrm{HR}^{\star}$ & 1.00 & 0.88 & $0.72,1.08$ & 0.81 & $0.66,1.01$ & 0.85 & $0.69,1.05$ & $0 \cdot 138$ \\
\hline Multivariable HR† & 1.00 & 0.89 & $0.72,1.10$ & 0.82 & $0.66,1.03$ & 0.87 & $0.70,1 \cdot 10$ & 0.299 \\
\hline Total CVD & & & & & & & & \\
\hline Number & 605 & & 526 & & 506 & & 06 & \\
\hline Age- and sex-adjusted HR & 1.00 & 0.85 & $0.76,0.95$ & 0.82 & $0.73,0.93$ & 0.80 & $0.71,0.89$ & $<0.001$ \\
\hline Multivariable $\mathrm{HR}^{\star}$ & 1.00 & 0.90 & $0.80,1.01$ & 0.90 & $0.80,1.02$ & 0.88 & $0.78,0.99$ & 0.069 \\
\hline Multivariable $\mathrm{HR} \dagger$ & 1.00 & 0.93 & $0.82,1.05$ & 0.95 & $0.83,1.08$ & 0.96 & $0.84,1.10$ & 0.835 \\
\hline All causes & & & & & & & & \\
\hline Number & 1983 & & 1786 & & 1745 & & 92 & \\
\hline Age- and sex-adjusted HR & 1.00 & 0.89 & $0.84,0.95$ & 0.90 & $0.84,0.95$ & 0.90 & $0.85,0.96$ & 0.007 \\
\hline Multivariable $\mathrm{HR}^{\star}$ & 1.00 & 0.93 & $0.87,0.99$ & 0.96 & $0.9, \quad 1.02$ & 0.97 & $0.91,1.04$ & 0.762 \\
\hline Multivariable HR† & 1.00 & 0.95 & $0.89,1.02$ & 0.99 & $0.93,1.06$ & 1.03 & $0.96,1.10$ & 0.188 \\
\hline
\end{tabular}

*The same variables as shown in the footnote of Table 2 .

†Adjusted further for fruit and bean intakes.

adjustment for them is justified. Further adjustment for other plant-based foods was also conducted for another multivariable model. A test for trend was used to assess statistical significance across exposure categories by including ordinal terms for each of the four categories and entering the variable as a continuous term in the model. A test for effect modification by sex was conducted using an interaction term generated by multiplying the fruit, vegetable and bean intakes by sex. A $P$ value of $<0.05$ was considered to be significant.

\section{Results}

Among the 25206 men and 34279 women followed up for an average of 12.7 years, 1207 men and 1036 women died from CVD, and 4514 men and 3029 women died from all causes. The deaths among men included 559 from stroke (128 intraparenchymal haemorrhages, 52 subarachnoid haemorrhages and 214 ischaemic strokes) and 258 from CHD. The respective numbers of deaths among women were 494 (104, 109 and 148) and 194.
Table 1 shows selected cardiovascular risk factors by fruit, vegetable and bean quartile. The participants with higher fruit intake smoked less, had lower mean ethanol intake, walked more and had higher education. The participants with higher vegetable intake were older, smoked less, had lower mean ethanol intake, walked more and had higher education. The participants with higher bean intake were older, smoked less, walked more and had higher education. The participants with higher intakes of fruit, vegetable and bean had higher mean intakes of total energy, cholesterol, $n-3$ fatty acids and sodium.

The associations of the fruit, vegetable and bean intakes with mortality from stroke, CHD, total CVD and all causes did not vary by sex $(P$ for interaction $>0.05)$. Thus, we combined men and women in the present study. Table 2 shows the sex- and age-adjusted and multivariable HR of mortality from stroke, CHD and total CVD, as well as total mortality according to quartiles of fruit intake. There were inverse associations of fruit intake with age- and sex-adjusted mortality from total stroke, haemorrhagic stroke, total CVD and total mortality. After adjustment for cardiovascular risk 
Table 4. Risk of mortality from stroke, CHD, total CVD and all causes according to quartiles of the frequency of bean intake (Hazard ratio (HR) values and $95 \% \mathrm{Cl}$ )

\begin{tabular}{|c|c|c|c|c|c|c|c|c|}
\hline & \multicolumn{7}{|c|}{ Quartiles of bean intake } & \multirow[b]{3}{*}{$P$ for trend } \\
\hline & \multirow[b]{2}{*}{ Q1 } & \multicolumn{2}{|r|}{ Q2 } & \multicolumn{2}{|r|}{ Q3 } & \multicolumn{2}{|c|}{ Q4 } & \\
\hline & & $\mathrm{HR}$ & $95 \% \mathrm{Cl}$ & $\mathrm{HR}$ & $95 \% \mathrm{Cl}$ & $\mathrm{HR}$ & $95 \% \mathrm{Cl}$ & \\
\hline Person-years & 191279 & \multicolumn{2}{|r|}{198098} & \multicolumn{2}{|r|}{156448} & \multicolumn{2}{|c|}{210229} & \\
\hline \multicolumn{9}{|l|}{ Total stroke } \\
\hline Number & 238 & \multicolumn{2}{|r|}{266} & \multicolumn{2}{|r|}{261} & \multicolumn{2}{|c|}{288} & \\
\hline Age- and sex-adjusted HR & 1.00 & 1.01 & $0.85,1 \cdot 21$ & 1.06 & $0.89,1.26$ & 0.86 & $0.73,1.02$ & 0.046 \\
\hline Multivariable $\mathrm{HR}^{*}$ & 1.00 & 1.00 & $0.84,1.19$ & $1 \cdot 10$ & $0.92,1.32$ & 0.90 & $0.75,1.08$ & $0 \cdot 188$ \\
\hline Multivariable HR $\dagger$ & 1.00 & 1.02 & $0.85,1.22$ & $1 \cdot 14$ & $0.95,1.38$ & 0.95 & $0.79,1.16$ & 0.496 \\
\hline \multicolumn{9}{|l|}{ Haemorrhagic stroke } \\
\hline Number & 88 & \multicolumn{2}{|r|}{100} & \multicolumn{2}{|r|}{99} & \multicolumn{2}{|c|}{106} & \\
\hline Age- and sex-adjusted HR & 1.00 & 1.05 & $0.79,1.40$ & $1 \cdot 16$ & $0.87,1.55$ & 0.90 & $0.68,1.20$ & 0.400 \\
\hline Multivariable $\mathrm{HR}^{*}$ & 1.00 & 1.06 & $0.80,1.42$ & 1.26 & $0.94,1.69$ & 1.03 & $0.77,1.40$ & 0.857 \\
\hline Multivariable HR† & 1.00 & $1 \cdot 10$ & $0.82,1.47$ & 1.34 & $0.98,1.82$ & $1 \cdot 11$ & $0.80,1.52$ & 0.620 \\
\hline \multicolumn{9}{|l|}{ Ischaemic stroke } \\
\hline Number & 85 & \multicolumn{2}{|r|}{90} & \multicolumn{2}{|r|}{84} & \multicolumn{2}{|c|}{103} & \\
\hline Age- and sex-adjusted HR & 1.00 & 0.96 & $0.71,1.29$ & 0.92 & $0.68,1.25$ & 0.85 & $0.63,1.13$ & 0.210 \\
\hline Multivariable $\mathrm{HR}^{*}$ & 1.00 & 0.94 & $0.69,1.26$ & 0.96 & $0.70,1.31$ & 0.88 & $0.64,1.19$ & 0.389 \\
\hline Multivariable $\mathrm{HR} \dagger$ & 1.00 & 0.95 & $0.70,1.29$ & 0.98 & $0.71,1.35$ & 0.92 & $0.66,1.26$ & 0.554 \\
\hline \multicolumn{9}{|l|}{$\mathrm{CHD}$} \\
\hline Number & 123 & \multicolumn{2}{|r|}{115} & \multicolumn{2}{|r|}{97} & \multicolumn{2}{|c|}{117} & \\
\hline Age- and sex-adjusted HR & 1.00 & 0.85 & $0.66,1.10$ & 0.78 & $0.59,1.01$ & $0 \cdot 70$ & $0.54,0.90$ & 0.006 \\
\hline Multivariable $\mathrm{HR}^{*}$ & 1.00 & 0.90 & $0.69,1.16$ & 0.85 & $0.65,1.12$ & $0 \cdot 80$ & $0.61,1.05$ & $0 \cdot 124$ \\
\hline Multivariable HR† & 1.00 & 0.93 & $0.72,1.21$ & 0.92 & $0.69,1.23$ & 0.88 & $0.66,1.18$ & 0.407 \\
\hline Other CVD & & & & & & & & \\
\hline Number & 195 & & 169 & & 175 & & & \\
\hline Age- and sex-adjusted HR & 1.00 & 0.78 & $0.64,0.96$ & 0.86 & $0.70,1.06$ & 0.72 & $0.59,0.88$ & 0.020 \\
\hline Multivariable $\mathrm{HR}^{\star}$ & 1.00 & 0.80 & $0.65,0.99$ & 0.93 & $0.75,1.15$ & 0.79 & $0.64,0.98$ & 0.097 \\
\hline Multivariable HR† & 1.00 & 0.82 & $0.66,1.01$ & 0.96 & $0.77,1.19$ & 0.82 & $0.65,1.02$ & 0.196 \\
\hline Total CVD & & & & & & & & \\
\hline Number & 556 & 550 & & & 533 & & & \\
\hline Age- and sex-adjusted HR & 1.00 & 0.90 & $0.80,1.01$ & 0.93 & $0.82,1.05$ & 0.78 & $0.69,0.87$ & $<0.001$ \\
\hline Multivariable $\mathrm{HR}^{*}$ & 1.00 & 0.91 & $0.81,1.02$ & 0.99 & $0.87,1.12$ & 0.84 & $0.74,0.95$ & 0.010 \\
\hline Multivariable HR $\dagger$ & 1.00 & 0.93 & $0.82,1.05$ & 1.03 & $0.91,1.17$ & 0.89 & $0.78,1.01$ & 0.106 \\
\hline All causes & & & & & & & & \\
\hline Number & 1883 & & 1868 & & 1741 & & & \\
\hline Age- and sex-adjusted HR & 1.00 & 0.92 & $0.86,0.98$ & 0.94 & $0.88,1.01$ & 0.86 & $0.80,0.91$ & $<0.001$ \\
\hline Multivariable $\mathrm{HR}^{*}$ & 1.00 & 0.94 & $0.88,1.00$ & 0.99 & $0.92,1.06$ & 0.90 & $0.84,0.96$ & 0.006 \\
\hline Multivariable HR† & 1.00 & 0.95 & $0.89,1.01$ & 1.00 & $0.94,1.08$ & 0.92 & $0.86,0.98$ & 0.025 \\
\hline
\end{tabular}

* The same variables as shown in the footnote of Table 2 .

† Adjusted further for fruit and vegetable intakes.

factors, these associations were attenuated slightly but remained statistically significant. The multivariable HR (95\% CI) of total stroke, haemorrhagic stroke, total CVD and total mortality in the highest $v$. lowest quartiles of fruit intake were $0.67(0.55,0.81, P$ for trend $<0.001), 0.63(0.46$, $0.87, \quad P$ for trend $=0.004), 0.75 \quad(0.66,0.85, \quad P$ for trend $<0.001)$ and $0.86(0.80,0.92, P$ for trend $<0.001)$. These inverse associations did not alter materially when we adjusted further for vegetable and bean intakes.

Table 3 shows the sex- and age-adjusted and multivariable HR according to quartiles of vegetable intake. Vegetable intake was inversely associated with sex- and age-adjusted mortality from CHD, total CVD and total mortality; after adjustment for cardiovascular risk factors, these associations were weakened but the association with CHD remained statistically significant, that with CVD was borderline statistically significant, but that with total mortality was no longer statistically significant. The multivariable HR (95\% CI) of CHD and total CVD in the highest $v$. lowest quartiles of vegetable intake were $0.77(0.58,1.00, P$ for trend $=0.08)$ and $0.88(0.78,0.99$, $P$ for trend $=0 \cdot 07)$.
Table 4 shows the sex- and age-adjusted and multivariable HR according to quartiles of bean intake. Bean intake was inversely associated with sex- and age-adjusted mortality from total stroke, CHD, other CVD, total CVD and total mortality. After adjustment for cardiovascular risk factors, these associations were weakened, and were no longer statistically significant except for other CVD, total CVD and total mortality. The respective multivariable $\mathrm{HR}$ in the highest $v$. lowest quartiles of bean intake were $0.79(0.64,0.98, P$ for trend $=0.10), 0.84(0.74,0.95, P$ for trend $=0.01)$ and 0.90 $(0.84,0.96, P$ for trend $=0.01)$. After further adjustment for fruit and vegetable intakes, these inverse associations became weak and were of borderline statistical significance.

\section{Discussion}

In the present large prospective study of Japanese men and women, we found inverse associations of plant-based food intake with mortality from CVD after adjustment for cardiovascular risk factors. High fruit intake was associated with reduced mortality from haemorrhagic and total stroke, total 
CVD and all causes; vegetable intake tended to be associated with reduced mortality from CHD, total CVD and all causes; bean intake was associated with reduced mortality from total CVD as well as total mortality.

Further adjustment for other plant-based foods did not alter the association of fruit intake with mortality, but attenuated the associations of vegetable and bean intakes with mortality. The weakened associations, however, do not necessarily negate potential protective effects of vegetable and bean intakes, because those intakes were moderately correlated with fruit intake: the Spearman correlation coefficients of vegetable and bean intakes were 0.36 and $0 \cdot 28$, respectively. It is possible that vegetable or bean intake is merely a surrogate for fruit intake in the present study.

The meta-analysis of eight cohort studies showed that vegetable and fruit intakes were associated with a reduced risk of stroke $^{(1)}$, and several Japanese cohort studies also showed that intakes of vegetables, fruits and soya were associated with a reduced risk of stroke ${ }^{(11,12,14)}$. The present study showed that intakes of fruits, but not vegetables and beans, were associated with a reduced risk of stroke.

The meta-analysis for studies of Western countries showed that vegetable, and fruit intakes were associated with a reduced risk of $\mathrm{CHD}^{(2)}$. The present study added the evidence on fruit intake and reduced mortality from CHD in Japanese.

A recent Japanese study reported that soya intake was associated with a reduced risk of ischaemic stroke and myocardial infarction $^{(14)}$. The present study extends the evidence that bean intake was associated with reduced mortality from total CVD and all causes.

As for the mechanisms for the inverse association between fruit intake and CVD, vitamin $\mathrm{C}$ reduces the lipid oxidation of LDL-cholesterol $^{(20)}$ and enhances the formation of endothelial prostacyclin that decreases vascular tone and inhibits platelet aggregation $^{(21)}$. Potassium, magnesium, calcium, fibre and folate exert the beneficial effects described previously ${ }^{(22-25)}$.

The protective effects of soyabean intake on CVD are now highlighted in Western countries, based on epidemiological studies that showed a lower incidence of CVD in Asian populations consuming soya foods as a dietary staple compared with those who consumed a typical Western diet ${ }^{(9,10,26,27)}$. Some clinical trials in Western countries that failed to detect the protective association led to the speculation that only high levels of habitual intake exerted the beneficial effects $^{(10,27)}$, and research into populations with a high level of intake was required. In the Japan Collaborative Cohort Study, we did not ask about the intake of soyabeans specifically, but the present finding of the inverse association between bean intake and mortality from total CVD would suggest a cardio-protective effect of soyabeans, because they are the most common beans eaten in Japan ${ }^{(28)}$.

There are several mechanisms for the inverse association between bean intake and CVD. Potassium, calcium and fibre, which are plentiful in beans, may play a role in lowering blood pressure ${ }^{(23,24)}$. Potassium also inhibits platelet aggregation $^{(23)}$, and fibre, isoflavones, soya protein and saponins help lower total cholesterol levels ${ }^{(9,24)}$. Isoflavones also enhance antioxidant activity and improve arterial stiffness $^{(9,25)}$. Folate, which is also plentiful in beans, lowers serum homocysteine levels, a correlate for arterial endothelial dysfunction $^{(25)}$.
Some limitations warrant discussion. First, the food frequency questionnaire used in the present study had high reproducibility but low-to-moderate validity for the estimation of the fruit, vegetable and bean intakes. Thus, some non-differential misclassification would be to weaken the diet-disease association. Second, a number of subjects were excluded because they did not respond sufficiently to the FFQ. However, a potential selection bias may be small because of no difference in mortality and cardiovascular risk characteristics between persons who responded to the food frequency questionnaire and those who did not.

Healthy behaviours associated with plant-based food intake might confound the association with mortality from CVD. Non-smoking, appropriate alcohol intake, more physical activity and higher education are potential confounders in the present study. However, after adjustment for these confounding variables, the associations with mortality from CVD remained statistically significant, suggesting that independent effects of plant-based foods exist for the prevention of CVD. Residual confounding and the contribution of other unexamined factors, however, were not negated.

In conclusion, fruit intake was inversely associated with mortality from stroke, total CVD and all causes, and bean intake was also inversely associated with mortality from total CVD and all causes among Japanese men and women. The present findings suggest the potential for beneficial effects of plant-based food intake for the prevention of CVD in general populations.

\section{Acknowledgements}

J. N. participated in the study analysis, interpretation of data, drafting of the manuscript and provided statistical expertise. K. M. participated in the study analysis and interpretation of data. H. I., Y. W., C. D., H. T., A. Y., S. K., A. K., T. K., Y. W., Y. I. and A. T. participated in the study concept and design, acquisition of data and interpretation of data, and critical revision of the manuscript. The authors thank all staff members involved in the present study for their valuable help in conducting the baseline survey and follow-up. The JACC Study group investigators are: Dr Akiko Tamakoshi (present chairperson of the study group), Aichi Medical University School of Medicine; Dr Mitsuru Mori, Sapporo Medical University School of Medicine; Dr Yutaka Motohashi, Akita University School of Medicine; Dr Ichiro Tsuji, Tohoku University Graduate School of Medicine; Dr Yosikazu Nakamura, Jichi Medical School; Dr Hiroyasu Iso, Osaka University Graduate School of Medicine; Dr Haruo Mikami, Chiba Cancer Center; Dr Yutaka Inaba, Juntendo University School of Medicine; Dr Yoshiharu Hoshiyama, University of Human Arts and Sciences; Dr Hiroshi Suzuki, Niigata University School of Medicine; Dr Hiroyuki Shimizu, Gifu University School of Medicine; Dr Hideaki Toyoshima, Nagoya University Graduate School of Medicine; Dr Kenji Wakai, Aichi Cancer Center Research Institute; Dr Shinkan Tokudome, Nagoya City University Graduate School of Medical Sciences; Dr Yoshinori Ito, Fujita Health University School of Health Sciences; Dr Shuji Hashimoto, Fujita Health University School of Medicine; Dr Shogo Kikuchi, Aichi Medical University School of Medicine; Dr Akio Koizumi, 
Graduate School of Medicine and Faculty of Medicine, Kyoto University; Dr Takashi Kawamura, Kyoto University Center for Student Health; Dr Yoshiyuki Watanabe, Kyoto Prefectural University of Medicine Graduate School of Medical Science; Dr Tsuneharu Miki, Graduate School of Medical Science, Kyoto Prefectural University of Medicine; Dr Chigusa Date, Faculty of Human Life and Environment, Nara Women's University; Dr Kiyomi Sakata, Wakayama Medical University; Dr Takayuki Nose, Tottori University Faculty of Medicine; Dr Norihiko Hayakawa, Research Institute for Radiation Biology and Medicine, Hiroshima University; Dr Takesumi Yoshimura, Fukuoka Institute of Health and Environmental Sciences; Dr Akira Shibata, Kurume University School of Medicine; Dr Naoyuki Okamoto, Kanagawa Cancer Center; Dr Hideo Shio, Moriyama Municipal Hospital; Dr Yoshiyuki Ohno, Asahi Rosai Hospital; Dr Tomoyuki Kitagawa, Cancer Institute of the Japanese Foundation for Cancer Research; Dr Toshio Kuroki, Gifu University; Dr Kazuo Tajima, Aichi Cancer Center Research Institute. The Japan Collaborative Cohort Study was supported by grants-in-aid for scientific research from the Ministry of Education, Science, Sports and Culture of Japan Monbusho (61010076, 62010074, 63010074, 1010068, 2151065, 3151064, 4151063, 5151069, $6279102,11181101,17015022$ and 18074011). None of the authors had any conflicts of interest.

\section{References}

1. He FJ, Nowson CA \& MacGregor GA (2006) Fruit and vegetable consumption and stroke: meta-analysis of cohort studies. Lancet 367, 320-326.

2. Dauchet L, Amouyel P, Hercberg S, et al. (2006) Fruit and vegetable consumption and risk of coronary heart disease: a metaanalysis of cohort studies. J Nutr 136, 2588-2593.

3. Fraser GE, Sabate J, Beeson WL, et al. (1992) A possible protective effect of nut consumption on risk of coronary heart disease: the Adventist Health Study. Arch Intern Med 152, 1416-1424.

4. Hu FB, Stampfer MJ, Manson JE, et al. (1998) Frequent nut consumption and risk of coronary heart disease in women: prospective cohort study. Br Med J 317, 1341-1345.

5. Ellsworth JL, Kushi LH \& Folsom AR (2001) Frequent nut intake and risk of death from coronary heart disease and all causes in postmenopausal women: the Iowa Women's Health Study. Nutr Metab Cardiovasc Dis 11, 372-377.

6. Albert CM, Gaziano JM, Willett WC, et al. (2002) Nut consumption and decreased risk of sudden cardiac death in the Physicians' Health Study. Arch Intern Med 162, $1382-1387$.

7. Kubo M, Kiyohara Y, Kato I, et al. (2003) Trends in the incidence, mortality, and survival rate of cardiovascular disease in a Japanese community: the Hisayama study. Stroke 34, $2349-2354$.

8. Sankai T, Miyagaki T \& Iso H (1991) A population-based study of the proportion by type of stroke determined by computed tomography scan. Nippon Koshu Eisei Zasshi 38, 901-909, (in Japanese).

9. Erdman JW Jr \& AHA Science Advisory (2000) Soy protein and cardiovascular disease: a statement for healthcare professionals from the Nutrition Committee of the AHA. Circulation 102, 2555-2559.

10. van der Schouw YT, Kreijkamp-Kaspers S, Peeters PH, et al. (2005) Prospective study on usual dietary phytoestrogen intake and cardiovascular disease risk in Western women. Circulation 111, 465-471.

11. Sauvaget C, Nagano J, Allen N, et al. (2003) Vegetable and fruit intake and stroke mortality in the Hiroshima/Nagasaki Life Span Study. Stroke 34, 2355-2360.

12. Yokoyama T, Date C, Kokubo Y, et al. (2000) Serum vitamin C concentration was inversely associated with subsequent 20 -year incidence of stroke in a Japanese rural community: The Shibata Study. Stroke 31, 2287-2294.

13. Takachi R, Inoue M, Ishihara J, et al. (2007) Fruit and vegetable intake and risk of total cancer and cardiovascular disease: Japan Public Health Center-Based Prospective Study. Am J Epidemiol 167, 59-70.

14. Kokubo Y, Iso H, Ishihara J, et al. (2007) Association of dietary intake of soy, beans, and isoflavones with risk of cerebral and myocardial infarctions in Japanese populations: the Japan Public Health Center-Based (JPHC) Study Chort I. Circulation 116, 2553-2562.

15. Nakamura K, Nagata C, Oba S, et al. (2008) Fruit and vegetable intake and mortality from cardiovascular disease are inversely associated in Japanese women but not in men. $J$ Nutr 138, $1129-1134$.

16. Iso H \& Kubota Y (2007) Nutrition and Disease in the Japan Collaborative Cohort Study for Evaluation of Cancer (JACC). Asian Pacific J Cancer Prev 8, 35-80.

17. Bazzano LA, He J, Ogden LG, et al. (2002) Fruit and vegetable intake and risk of cardiovascular disease in US adults: the first National Health and Nutrition Examination Survey Epidemiologic Follow-up Study. Am J Clin Nutr 76, 93-99.

18. Genkinger JM, Platz EA, Hoffman SC, et al. (2004) Fruit, vegetable and antioxidant intake and all-cause, cancer, and cardiovascular disease mortality in a community-dwelling population in Washington County, Maryland. Am J Epidemiol 160, $1223-1233$

19. Date C, Fukui M, Yamamoto A, et al. (2005) Reproducibility and validity of a self-administered food frequency questionnaire used in JACC Study. J Epidemiol 15, S9-23.

20. Gaziano JM (1999) Antioxidant vitamins and cardiovascular disease. Proc Assoc Am Physicians 111, 2-9.

21. Lefer AM (1990) Prostacyclin, high density lipoproteins, and myocardial ischemia. Circulation 81, 2013-2015.

22. He FJ \& MacGregor GA (2001) Fortnightly review: beneficial effects of potassium. Br Med J 323, 497-501.

23. Jenkins DJ, Kendall CW \& Vuksan V (2000) Viscous fibers, health claims, and strategies to reduce cardiovascular disease risk. Am J Clin Nutr 71, 401-402.

24. van der Schouw YT, Pijpe A, Lebrun CE, et al. (2002) Higher usual dietary intake of phytoestrogens is associated with lower aortic stiffness in postmenopausal women. Arterioscler Thromb Vasc Biol 22, 1316-1322.

25. Bazzano LA, He J, Ogden LG, et al. (2002) Dietary intake of folate and risk of stroke in US men and women: NHANES I Epidemiologic Follow-up Study: National Health and Nutrition Examination Survey. Stroke 33, 1183-1188.

26. Kreijkamp-Kaspers S, Kok L, Grobbee DE, et al. (2004) Effect of soy protein containing isoflavones on cognitive function, bone mineral density, and plasma lipids in postmenopausal women: a randomized controlled trial. J Am Med Assoc 292, $65-74$.

27. Sacks FM (2005) Dietary phytoestrogens to prevent cardiovascular disease: early promise unfulfilled. Circulation 111, 385-387.

28. Ministry of Health, Labour and Welfare, Japan (2003) The National Nutrition Survey in Japan, 2002. Tokyo: Daiichi-shuppan. 